\title{
Adaptação transcultural do instrumento para exame do pé diabético em 3 minutos
}

\author{
Cross-cultural adaptation of the diabetic foot examination instrument in 3 minutes
}

Adaptación transcultural del instrumento para el examen del pie diabético en 3 minutos

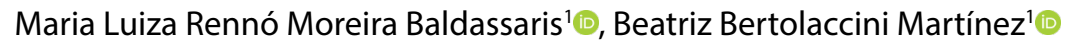 \\ 'Universidade do Vale do Sapucaí (UNIVAS), Pouso Alegre, Minas Gerais, Brasil
}

\section{Resumo}

Introdução: O "How to do a 3-minute diabetic foot exam", elaborado por Armstrong et al foi projetado para fornecer aos profissionais de saúde uma forma aprofundada, resumida e facilmente aplicável para avaliar os pés do paciente diabético na Atenção Primária à Saúde. Objetivo: Traduzir para a língua portuguesa, adaptar ao contexto cultural brasileiro e testar as propriedades de medidas deste instrumento. Métodos: Seguindo orientação padrão da literatura, o instrumento foi traduzido para o português, adaptado culturalmente e testado em relação à reprodutibilidade, validade de face, conteúdo e construto. Nas etapas de adaptação cultural e validação foram entrevistados 30 profissionais de saúde e 60 pacientes, respectivamente. Resultados: $O$ instrumento foi adaptado ao contexto linguístico e cultural da população mantendo todas as características essenciais do instrumento original em Inglês e sendo preservadas as equivalências idiomática, semântica, conceitual e cultural. Todos os itens do instrumento apresentaram concordância calculado pelo Índice de Validade de Conteúdo (IVC) > 0,9. O a de Cronbach foi de 0,67. O Coeficiente de Correlação Intraclasse interobservador foi de 0,73 (IC95\%:0,58-0,85) e intraobservador foi de 0,65 (IC95\%: 0,45-0,81), demostrando uma reprodutibilidade satisfatória. O instrumento e o teste do monofilamento apresentaram correlação positiva com significância estatística $(\rho=0,41 ; p<0,01)$. Conclusão: Este trabalho traduziu para a língua portuguesa, adaptou ao contexto cultural brasileiro e testou as propriedades de medidas do instrumento americano: "How to do a 3-minute diabetic foot exam".

Palavras-chave: Diabetes mellitus; Atenção Primária à Saúde; Pé diabético, Tradução.

\begin{abstract}
Background: The "How to do a 3-minute diabetic foot exam", developed by Armstrong et al., was designed to provide healthcare professionals with an in-depth, summarized, and easily applicable way to assess diabetic patients' feet in Primary Health Care. Objective: To translate into Portuguese, adapt to the Brazilian cultural context, and test the measurement properties of this instrument Methods: Following standard literature guidance, the instrument was translated into Portuguese, culturally adapted and tested for reproducibility, face validity, content, and construct. In the stages of cultural adaptation and validation, 30 health professionals and 60 patients, respectively, were interviewed. Results: The instrument was adapted to the linguistic and cultural context of the population maintaining all the essential characteristics of the original instrument in English and preserving the idiomatic, semantic, conceptual and cultural equivalences. All items of the instrument showed agreement calculated by the Content Validity Index $(\mathrm{CVI})>0.9$. Cronbach's a was 0.67. The interobserver Intraclass Correlation Coefficient was 0.73 (95\% Cl: 0.58-0.85) and intraobserver correlation was 0.65 (95\% Cl: $0.45-0.81)$, showing satisfactory reproducibility. The instrument and the monofilament test showed a positive correlation with statistical significance $(\rho=0.41 ; p<0.01)$. Conclusion: This work translated into Portuguese, adapted to the Brazilian cultural context, and tested the measurement properties of the American instrument: "How to do a 3-minute diabetic foot exam".
\end{abstract}

Keywords: Diabetes mellitus; Primary Health Care; Diabetic Foot, Translation.

Como citar: Baldassaris MLRM, Martínez BB. Adaptação transcultural do instrumento para exame do pé diabético em 3 minutos. Rev Bras Med Fam Comunidade. 2020, Jan-Dez; 15(42):2008. https://doi.org/10.5712/rbmfc15(42)2008

\author{
Autor correspondente: \\ Maria Luiza Rennó Moreira Baldassaris. \\ E-mail: malurenno@yahoo.com.br \\ Fonte de financiamento: \\ declaram não haver. \\ Parecer CEP: \\ Parecer de número 1.560.278 \\ (Faculdade de Ciências Médicas Dr. José \\ Antônio Garcia Coutinho), aprovado em \\ 25/05/2016. \\ Procedência e revisão por pares: \\ Não encomendado; revisão por pares \\ externa \\ Recebido em: 05/03/2019. \\ Aprovado em: 26/09/2019.
}




\section{Resumen}

Introducción: El "How to do a 3-minute diabetic foot exam" de Armstrong et al, fue diseñado para proporcionar a los profesionales de la salud una forma profundizada, resumida y fácilmente aplicable de evaluar los pies de los pacientes diabéticos en la atención primaria a la salud. Objetivo: Traducir al portugués, adaptarse al contexto cultural brasileño y probar las propiedades de medición de este instrumento. Métodos: Siguiendo la guía estándar de la literatura, el instrumento fue traducido al portugués, culturalmente adaptado y probado para su reproducibilidad, validez aparente, contenido y construcción. En las etapas de adaptación cultural y validación, se entrevistó a 30 profesionales de la salud y 60 pacientes, respectivamente. Resultados: El instrumento se adaptó al contexto lingüístico y cultural de la población manteniendo todas las características esenciales del instrumento original en inglés y preservando las equivalencias idiomáticas, semánticas, conceptuales y culturales. Todos los ítems del instrumento mostraron acuerdo calculado por el Índice de Validez del Contenido $(\mathrm{CVI})>0.9$. El a de Cronbach fue de 0,67. El Coeficiente de Correlación Intraclase interobservador fue de 0,73 (IC 95\%: 0,58-0,85) y la correlación intraobservador fue de 0,65 (IC 95\%: 0,45-0,81), que muestra una reproducibilidad satisfactoria. El instrumento y la prueba de monofilamento presentaron correlación positiva con significación estadística $(\rho=0,41 ; p<0,01)$. Conclusión: Este trabajo se tradujo al portugués, se adaptó al contexto cultural brasileño y probó las propiedades de medición del instrumento estadounidense: "How to do a 3-minute diabetic foot exam ".

Palabras clave: Diabetes mellitus; Atención Primaria a la Salud; Pie diabético, Traducción.

\section{Introdução}

No Brasil, estima-se que cerca de 12 a $13 \%$ da população adulta tenha diabetes mellitus (DM), ${ }^{1}$ prevalecendo em faixas etárias mais avançadas. Atinge em torno de $7 \%$ da população entre 30 e 69 anos e $18 \%$ das pessoas acima de 65 anos. $^{2}$

O pé diabético está entre as causas mais frequentes de complicações do DM. A neuropatia (lesão do nervo) nos pés, combinada com a diminuição do fluxo sanguíneo, aumenta a chance de úlceras, infecção e eventual necessidade de amputação do membro. ${ }^{3}$

Segundo a definição do Consenso Internacional sobre Pé Diabético, ${ }^{4}$ entende-se como pé diabético a infecção, a ulceração e/ou a destruição dos tecidos profundos associados a anormalidades neurológicas e a vários graus de doença vascular periférica nos membros inferiores.

O diagnóstico precoce das alterações decorrentes do DM, associado ao tratamento oportuno e ao bom cuidado com os pés, pode prevenir complicações, conforme comprovação da literatura. ${ }^{5,6}$ Já ficou demonstrado, também, que, das amputações e ulcerações, metade pode ser prevenida pelo diagnóstico precoce e tratamento adequado. ${ }^{7}$

Para diminuir a incidência de úlcera, a American Diabetes Association (ADA) recomenda que os pacientes com diabetes realizem os exames dos pés anualmente, identificando fatores de risco para úlcera e amputação. . $^{8}$

Apesar da avaliação cuidadosa do pé do diabético ser uma medida eficaz para prevenir úlceras, existem vários estudos que demonstram que o exame do pé é uma das atividades menos realizada no monitoramento do paciente diabético, principalmente na Atenção Primária à Saúde (APS).8,10

AAPS é o local ideal para o acompanhamento integral da pessoa com DM: ela é o nível de atenção mais próximo da população e é responsável pelo cuidado longitudinal, integral e coordenado de sua população de referência. ${ }^{11}$

A partir do dado de prevalência de DM, é possível a equipe estabelecer uma organização para lidar com este agravo. Para isso, é preciso que ela leve em conta seus recursos atuais para oferta do cuidado e que mapeie os demais pontos de cuidado que compõem a Rede de Atenção à Saúde, que poderão ser acionados a depender das necessidades da população atendida. ${ }^{11}$ 
Cabe aos profissionais de saúde a atenção na identificação das pessoas em risco para o DM e a intensificação de ações para promover seu controle entre os já diagnosticados. Ressalta-se a necessidade de avaliar os membros inferiores dos diabéticos de forma cuidadosa e com frequência regular, além de desenvolver atividades educativas para o paciente e sua família, visando o autocuidado e um bom controle glicêmico. ${ }^{12,13}$ Também é importante superar as barreiras que dificultam a adoção do exame dos pés dos pacientes diabéticos pelos profissionais de saúde. Dentre elas, destaca-se a grande demanda das equipes e pouco tempo para estas avaliações.

Estudos confirmam deficiências no rastreio do pé diabético e estratificação de risco. ${ }^{8}$ Por isso, é preciso medidas práticas para promover o exame do pé do paciente diabético, levando em consideração que programas de prevenção e cuidados com os pés são intervenções altamente eficazes em termos de custo para o sistema de saúde e para as pessoas com diabetes.

O objetivo deste trabalho foi traduzir para a língua portuguesa, adaptar ao contexto cultural brasileiro e testar as propriedades de medidas - reprodutibilidade e validade - do instrumento de triagem "How to do a 3-minute diabetic foot exam", para avaliação de pacientes diabéticos na Atenção Primária à Saúde.

\section{Métodos}

Trata-se de um estudo do tipo metodológico, fundamentado no referencial teórico de adaptação cultural e validação, ${ }^{14-17}$ realizado em Pouso Alegre, MG, na Universidade do Vale do Sapucaí (UNIVAS), que teve início após aprovação pelo Comitê de Ética em Pesquisa.

Miller et al., ${ }^{18}$ em 2004, elaboraram um instrumento chamado: "How to do a 3-minute diabetic foot exam". Eles procuraram fornecer aos profissionais de saúde uma forma aprofundada e facilmente aplicável à prática diária para avaliar a extremidade inferior do paciente diabético. Este exame consiste em três componentes: Item 1 - a história do paciente, Item 2 - um exame físico e Item 3 - educação do paciente. Cada um dos componentes sendo realizado em 1 minuto, levando menos tempo do que o exame abrangente ${ }^{19}$ (recomendado pela ADA para especialistas), eliminando barreiras comuns para avaliações frequentes.

Além disso, os autores apresentam uma tabela de risco (Item 4) com as devidas indicações e o tempo para serem reavaliadas ou encaminhadas para o especialista, de acordo com as alterações encontradas.

Após autorização do autor David G. Armstrong (via e-mail), viabilizou-se o desenvolvimento inicial do projeto.

Seguindo as orientações propostas por Guillemin et al. ${ }^{15}$ e Beaton et al. ${ }^{14} \mathrm{o}$ instrumento desenvolvido por Miller et al.:18 "How to do a 3-minute diabetic foot exam", em sua versão original, foi traduzido da língua inglesa para a língua portuguesa por dois tradutores (T1 e T2) independentes. Foram dois tradutores brasileiros com conhecimento avançado na língua inglesa. Apenas um dos tradutores foi informado sobre os objetivos do estudo, de modo a atingir uma tradução conceitual e não estritamente literal.

As duas traduções foram analisadas por um grupo multidisciplinar composto por dois metodologistas, dois tradutores e profissionais especialistas da área da saúde, incluindo quatro médicos endocrinologistas e um médico de família, além de um enfermeiro especializado em estomatoterapia. 
Esta equipe analisou e comparou as versões ao instrumento original. Foram levantadas as diferenças e discrepâncias originadas no processo de tradução. Todas as divergências foram discutidas até a concordância quanto ao significado original das frases e mantiveram-se as características originais (validação semântica). Por consenso, foi criada uma versão traduzida e adaptada da versão original do instrumento, baseada em elementos das duas versões iniciais (versão $T_{1,2}$ ).

$A$ versão $T_{1,2}$ foi traduzida novamente para a língua inglesa (back-translation) por dois tradutores independentes, que não conheciam o instrumento original e os objetivos do trabalho. As duas versões retraduzidas foram analisadas e comparadas ao instrumento original pelo mesmo grupo multidisciplinar, buscando discutir diferenças e discrepâncias originadas do processo. Novamente, por consenso, chegouse à versão retraduzida $\left(B T_{1,2}\right)$. A versão $B T_{1,2}$ foi enviada para o autor do instrumento original (via e-mail). Ele avaliou e confirmou a manutenção das características essenciais do texto original. Com isso, a versão $\mathrm{T}_{1,2}$ passou a ser considerada a versão Pré-Final e foi utilizada para adaptação cultural.

$\mathrm{Na}$ fase de adaptação cultural a população-alvo avaliou a compreensão e a relevância de cada afirmativa do instrumento. Para compor esta população, foram selecionados 30 profissionais de saúde que estão presentes na atenção primária e mantêm um contato direto com os pacientes diabéticos na comunidade. Para isso, foram considerados os seguintes critérios de inclusão: ser médico(a) ou enfermeiro(a) da Atenção Primária à Saúde dos municípios estudados (Pouso Alegre e Congonhal, MG) e aceitar o Termo de Consentimento Livre e Esclarecido. Esta fase foi importante para reconhecer as adequações que se fizessem necessárias de acordo com suas avaliações. Foi realizada através de ambiente virtual (Google Forms), sendo enviado um e-mail para cada profissional com o link do Termo de Consentimento Livre e Esclarecido e da entrevista, após um contato prévio via telefone.

Após cada pergunta, os profissionais foram convidados a explicar a questão da forma que entenderam (segundo suas próprias palavras), sugerir mudanças na formulação da mesma (adaptação da questão) e descrever o quanto estaria relacionada com a avaliação da prevenção de complicações do diabetes.

Os dados da entrevista foram colhidos e comparados, incluindo as adaptações necessárias para adequada compreensão dos pacientes. Esta versão final foi considerada traduzida e adaptada culturalmente.

Na fase de validação foram avaliados 60 pacientes no período estabelecido obedecendo aos seguintes critérios de elegibilidade:

- Critérios de Inclusão: Pacientes atendidos em Unidades de Atenção Primária à Saúde; Pacientes com diagnóstico de Diabetes tipo 1 e 2; Pacientes maiores de 18 anos.

- Critérios de Não Inclusão: Pacientes com grandes amputações de ambos os membros inferiores; Pacientes que não assinaram o Termo de Consentimento.

Nesta etapa foram testadas a reprodutibilidade $(n=30)$ e a validade de construto $(n=60)$.

Foram 90 participantes no total, sendo 30 na fase pré-teste (adaptação cultural), conforme as diretrizes para o processo de adaptação transcultural, ${ }^{14}$ além de 30 pacientes seguindo as diretrizes propostas por Mokkink et al., ${ }^{20}$ para testar a confiabilidade intra e inter-avaliadores, e mais 30 pacientes de acordo com as orientações de Terwee et al., ${ }^{21}$ para testar a conformidade do instrumento.

A pesquisa foi realizada entre janeiro e dezembro de 2016. As avaliações dos pacientes foram realizadas por uma médica de família e comunidade (MFC) e dois residentes de Medicina de Família e Comunidade na Unidade de Atenção Primária à Saúde do bairro São João do município de Pouso Alegre. 
Todos os 60 pacientes foram avaliados pela MFC, sendo que 30 foram avaliados 2 vezes com intervalo de 1 semana e também foram avaliados pelas residentes. A Figura 1 apresenta um fluxograma contendo as etapas de execução do estudo.

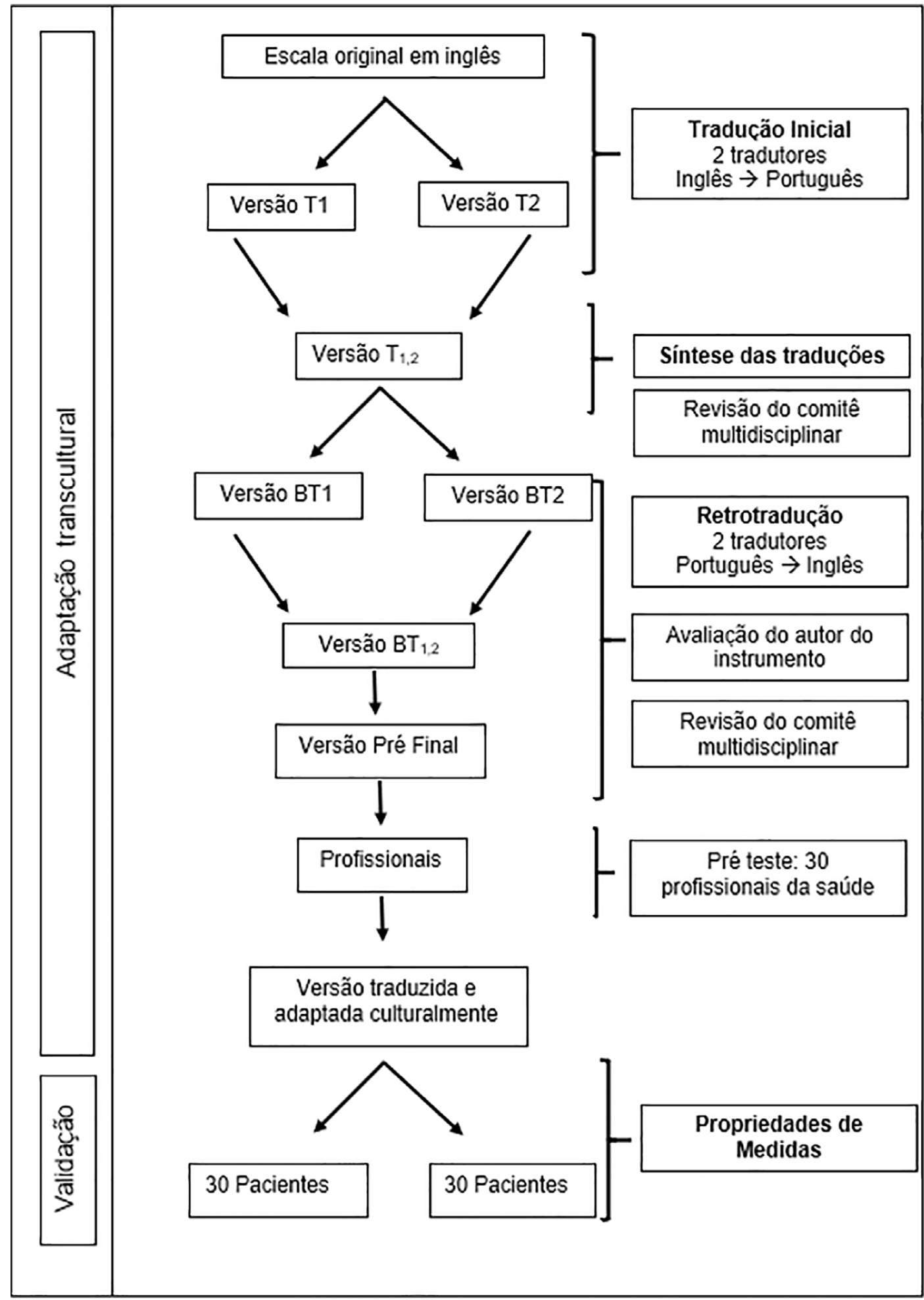

Figura 1. Fluxograma das etapas de execução do estudo. 
A confiabilidade do instrumento foi medida através do a de Cronbach analisando a consistência interna, quando os itens de um mesmo instrumento medem um mesmo construto. ${ }^{22}$

Na reprodutibilidade observou-se a correlação entre os dados obtidos quando o instrumento é aplicado em tempos diferentes (reprodutibilidade intraobservador), ou por observadores diferentes (reprodutibilidade inter-observadores).

A validade de um instrumento é definida pela capacidade de medir aquilo que se propõe a medir e pode ser classificada em validade de face, de conteúdo e de construto. ${ }^{22}$

A validade de face verifica se o instrumento mede aquilo para o qual foi projetado. Ela foi determinada, por consenso, pela equipe multidisciplinar que participou da elaboração da versão em Português.

A validade de conteúdo diz respeito à relevância de cada item do instrumento para a mensuração do tema abordado. Também é avaliada pela mesma equipe. O instrumento dividido em 4 itens ("O que perguntar", "O que procurar", "O que ensinar" e "Necessita de um especialista") foi submetido aos entrevistados (30 profissionais de saúde) juntamente com um questionário de avaliação em que responderam se os itens eram: 1) não representativos; 2) necessitam de grande revisão para serem representativos; 3) necessitam de pequena revisão para serem representativos; 4) representativos. Foi calculado o Índice de Validade de Conteúdo (IVC) de cada um dos itens, sendo que o IVC é a soma das respostas 3) e 4) dividida pelo total de respostas. ${ }^{16}$

A validade de construto foi testada por meio da comparação do instrumento com um instrumento que avaliasse o mesmo tema. Para isso, foram selecionados os instrumentos: Escore de Sintomas Neuropáticos (ESN) e Escore de comprometimento Neuropático (ECN). Tais escores, de forma sistematizada, avaliam, respectivamente, os sintomas e os sinais neurológicos apresentados pelo paciente. ${ }^{23}$ Eles são validados e utilizados em pesquisas científicas. Além disso, identificam a presença de Neuropatia Diabética, por isso, foram escolhidos para serem utilizados e comparados ao instrumento estudado.

A validade de construto foi obtida pelo seguinte teste de correlação: Spearman para variáveis categóricas e não paramétricas.

Para todos os testes estatísticos, foi adotado um nível de significância de $5 \%$ e as análises estatísticas foram realizadas utilizando-se o software estatístico SPSS 20.0. e STATA 12.

Apenas participaram desse estudo os pacientes diabéticos e os profissionais que concordaram e assinaram o Termo de Consentimento Livre e Esclarecido. O estudo só foi realizado após a aprovação do Comitê de Ética em Pesquisa da Universidade do Vale do Sapucaí, de acordo com a Resolução $n^{\circ} 466$, de 12 de dezembro de 2012, sob o parecer de número 1.560.278.

\section{Resultados}

Foram realizadas as seguintes adaptações no instrumento traduzido: retirada de palavras redundantes como "anteriormente" e "previamente" no histórico do paciente. Troca da palavra "curada" por "cicatrizada" ao se referir à ferida. Retirada do termo "uso de nicotina" e manutenção de "tabagismo". Adequação do termo "ardência" por "queimação no pés". Inclusão do termo "rachaduras" ao se referir às fissuras, além da descrição de maceração interdigital, "pele esbranquiçada e mole". 
Adequações no cronograma de encaminhamento também foram realizadas: na prioridade "urgente" adequou-se para "encaminhamento para urgência (no mesmo dia)" ao invés de apenas "imediato". Nas demais categorias, meses foram tranformados em semanas, como no exemplo: "Consulta no prazo de 1 mês" para "encaminhamento em até 4 semanas".

Como o instrumento de origem é americano, os EUA possui um especialista em pés, que é médico (podiatric surgeon). Porém, no Brasil, não temos esta especialidade médica. Ainda na fase de adaptação cultural, foi utilizado material do Ministério da Saúde ${ }^{11}$ para ajudar na definição dos profissionais que realizariam as avaliações de acompanhamento.

O produto deste trabalho (instrumento traduzido e validado) foi publicado no repositório OSF e encontra-se disponível pelo link: https://osf.io/zfu64/ ou DOI: 10.17605/OSF.IO/ZFU64. ${ }^{24}$

Na fase de Adaptação Cultural dos 30 profissionais de saúde, 18 (60\%) eram médicos e $12(40 \%)$ eram enfermeiros. As características sociodemográficas dos profissionais de saúde estão descritas na Tabela 1.

Todos os profissionais entrevistados responderam que atendem pacientes diabéticos. Dos entrevistados, $21(70 \%)$ disseram não encontrar dificuldade em realizar o exame do pé do diabético e 9 (30\%) apontaram dificuldade na realização deste exame. Um instrumento pode sistematizar e facilitar esta avaliação para estes que apresentam dificuldade, principalmente pela grande demanda atendida nas unidades.

Para verificar a validade de novos instrumentos de uma forma geral, recomenda-se uma concordância de 0,90 ou mais do Índice de Validade de Conteúdo (IVC). ${ }^{16}$ Todos os itens do instrumento apresentaram essa concordância (IVC=1,0; IVC=0,96; IVC=0,96; IVC=0,93, respectivamente, aos itens de 1 a 4).

As características sociodemográficas dos pacientes que participaram da fase de validação foram descritas na Tabela 2.

Nas avaliações de reprodutibilidade observou-se a correlação entre os dados obtidos quando o instrumento é aplicado em tempos diferentes (reprodutibilidade intraobservador), ou por observadores diferentes (reprodutibilidade interobservadores). Para isto, utilizou-se o Coeficiente de Correlação Intraclasse (CCl). CCI (intraobservador): 0,73 (IC95\%=0,58-0,85), p<0,01 e CCl (inter-observador): 0,65 $(\mathrm{IC} 95 \%=0,45-0,81), p<0,01$.

Tabela 1. Caracterização sociodemográfica da população estudada na fase de adaptação cultural (Profissionais de Saúde), Pouso Alegre e Congonhal, 2016.

\begin{tabular}{ll}
\hline \multicolumn{1}{c}{ VARIÁVEIS } & \multicolumn{1}{c}{ VALORES (N=30) } \\
\hline Idade em anos & $37,3( \pm 9,19)$ \\
\hline Média $( \pm \mathrm{DP})$ & 34 \\
Mediana & \\
\hline Sexo & $22(73 \%)$ \\
\hline Feminino & $8(27 \%)$ \\
Masculino & \\
\hline Tempo de atuação na profissão & 0 \\
\hline$<1$ ano & $9(30 \%)$ \\
De 1 a 5 anos & $21(70 \%)$ \\
$>5$ anos (\%) &
\end{tabular}

$\mathrm{DP}=$ desvio padrão 
Tabela 2. Caracterização sociodemográfica da população estudada na fase de validação (pacientes), Pouso Alegre, 2016.

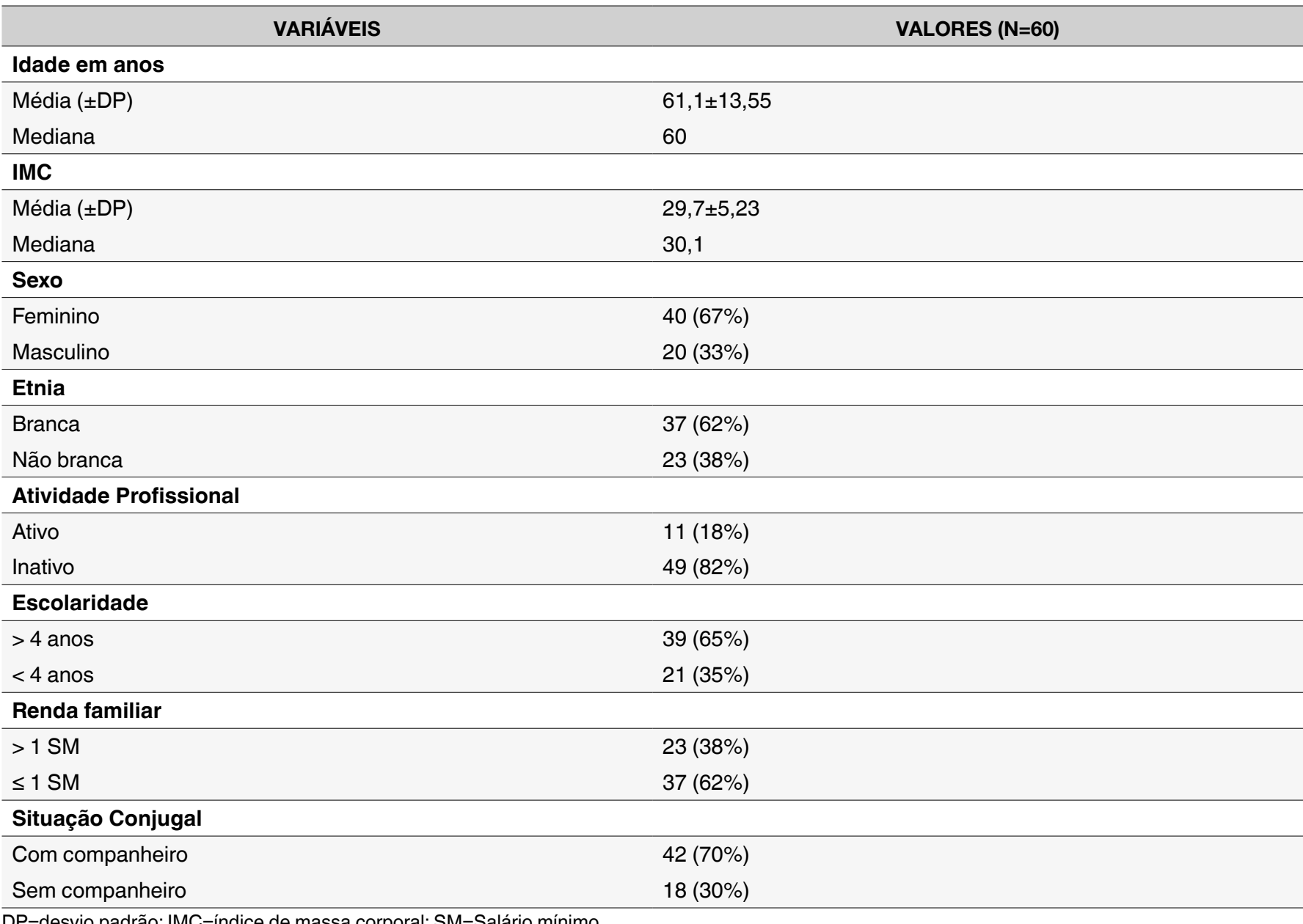

$\mathrm{DP}=$ desvio padrão; IMC=índice de massa corporal; $\mathrm{SM}=$ Salário mínimo

A Consistência Interna do instrumento foi analisada pelo $\alpha$ de Cronbach $(\alpha=0,67)$. Este resultado foi considerado adequado, visto que a literatura interpreta valores maiores que 0,60 como satisfatórios. ${ }^{22}$

A Validade de Construto foi verificada por meio da comparação do instrumento com o Escore de Sintomas Neuropáticos (ESN) associado ao Escore de Comprometimento Neuropático (ECN). Também foi feita a comparação com o Teste do monofilamento. Identificou-se correlação de Spearman positiva fraca, entre o instrumento em estudo e a presença de Neuropatia Periférica - $E S N+E C N(\rho=0,22 ; p=0,08)$, porém, sem significância estatística. Já com o teste do monofilamento apresentou correlação de Spearman positiva e nível de significância maior $>5 \%(\rho=0,41 ; p<0,01)$.

\section{Discussão}

Armstrong e colegas ${ }^{18}$ desenvolveram esta ferramenta com informações de alta qualidade de uma forma baseada em evidências de fácil incorporação em exames e avaliações na rotina do serviço. Ideal para Atenção Primária à Saúde (APS), a porta de entrada do sistema de saúde, onde se faz acompanhamento integral e longitudinal das pessoas além da coordenação do cuidado. Para alcançar alta resolutividade, a APS deve incorporar em sua rotina o cuidado dos pés das pessoas com diabetes. ${ }^{11}$ 
Foi proposto traduzir o instrumento de acordo com os padrões internacionais ${ }^{14,15}$ para tentar assegurar a disponibilidade de uma ferramenta adequada para uso na prática clínica atendendo à necessidade de avaliar os pacientes quanto ao risco de complicação do pé diabético. O objetivo principal do processo foi preservar a equivalência semântica e o conteúdo da versão original, minimizando possíveis erros que poderiam surgir de traduções imprecisas.

Adaptações linguísticas foram realizadas pelos participantes da equipe multidisciplinar. Com processo de consenso consegue-se que a tradução não sofra influências externas e viés individual sempre que possível. ${ }^{25}$

As adaptações culturais realizadas durante a fase de pré-teste envolveram adequações de termos e sugestões para melhor compreensão do instrumento. As sugestões foram bem interessantes, dentre elas destacam-se: explicação de como se faz o teste de toque Ipswich, explicação sobre o significado de Charcot, adequação dos termos "hipoglicemiantes" para "comprimidos", "úlcera" para "ferida". Também foi muito interessante o apontamento sobre criar ilustrações para contribuir para a educação em saúde dos pacientes, levando em consideração que algumas das pessoas atendidas em Equipes de Atenção Primária à Saúde apresentam baixa escolaridade (35\% neste estudo).

O Teste de Toque Ipswich é um teste neurológico alternativo que requer apenas o dedo indicador do examinador. Ele é realizado pedindo ao paciente para fechar os olhos enquanto o examinador coloca levemente o dedo indicador no primeiro, terceiro e quinto dedos do paciente por 2 segundos. O paciente é instruído a responder "sim" quando sentir o toque do examinador. Este método tem sido realizado por apresentar acurácia semelhante ao teste do monofilamento de Semmes-Weinstein para detectar perda da sensibilidade protetora ${ }^{26}$ e não requer a utilização de qualquer dispositivo específico.

Conforme o Manual do pé diabético do Ministério da Saúde, ${ }^{11}$ a periodicidade para avaliação dos pés do paciente diabético deverá ser anual quando não apresentar alterações. Quando isso não ocorrer, a reavaliação deve ser mais frequente. Esta avaliação deve conter no mínimo: anamnese cuidadosa, com pesquisa de fatores de risco e complicações; exame físico com pesquisa de úlceras, deformidades e outras alterações; além de exame neurológico e vascular. Na suspeita de Doença Vascular Periférica, o paciente deve ser encaminhado para avaliação vascular complementar (cirurgião vascular). Isso corrobora para que o instrumento deste estudo ajude nas avaliações dos pacientes diabéticos na APS, pois atende a todas estas necessidades.

O instrumento do estudo serve para identificar alterações nos pacientes diabéticos avaliados que possam classificá-lo de acordo com a prioridade de acompanhamento e necessidade de avaliação com o especialista. Qualquer alteração encontrada permite um direcionamento deste paciente para uma cuidado adequado.

$\mathrm{Na}$ análise do estudo, a reprodutibilidade do instrumento foi verificada através das avaliações de 30 pacientes. Segundo Pinto et al. ${ }^{27}$ os valores satisfatórios para $\mathrm{CCl}$ variam entre 0,4 e 0,75. Acima de 0,75, a reprodutibilidade é considerada excelente. Com isso, os valores encontrados do $\mathrm{CCl}$ intraobservador e inter-observador demonstram esta reprodutibilidade satisfatória. Apesar da imprecisão desta estimativa, o intervalo de confiança mostra que os valores encontrados em $95 \%$ da amostra são considerados satisfatórios ou excelentes. 
A validade de face foi determinada por consenso, a partir dos pareceres da equipe multidisciplinar, responsável pela versão brasileira da escala, durante a adaptação cultural. Durante o pré-teste ou adaptação cultural, os 30 profissionais de saúde determinaram a relevância de cada item da escala e realizaram a validade de face e conteúdo.

Foram encontradas algumas limitações na realização desta pesquisa. A pesquisa foi realizada na fase pré-teste em dois municípios da região do sul de Minas Gerais, para se obter o número adequado de profissionais de saúde. Já a avaliação dos pacientes foi realizada em apenas uma unidade de Atenção Primária à Saúde. Em estudos futuros pode-se avaliar pessoas de diferentes localidades, para se obter um maior rigor metodológico. Além disso, pode-se considerar que a amostra foi pequena e que o instrumento não envolve escores (soma de pontos), o que dificultou a fase de validação (comparando com outro instrumento já existente e validado que soma pontos).

Os escores (ESN e ECN) e o teste do monofilamento, utilizados na fase de validade, avaliam exclusivamente a neuropatia e não avaliam a vasculopatia. Mesmo sabendo que a neuropatia periférica é uma das lesões precursoras do pé diabético, a comparação entre os dois instrumentos mostrou uma Correlação de Spearman baixa. Porém, com o teste do monofilamento a correlação foi positiva fraca, e apresentou significância estatística, demonstrando a importância do instrumento para triagem de neuropatia.

O Item 3 do instrumento, relacionado às orientações a serem feitas aos pacientes, não pôde ser verificado e validado, pois exigiria um acompanhamento longitudinal dos pacientes para observar mudanças com as orientações fornecidas. Esta seria uma perspectiva futura de trabalho, levando em consideração que estudos têm relatado que as complicações e a utilização de serviços de saúde de urgência diminuem quando as pessoas recebem apoio educacional em comparação com aqueles que não recebem. ${ }^{28}$

Entretanto, o item 3 do instrumento mostra-se como uma ferramenta importante de educação em saúde aos pacientes, lembrando que esta parte foi traduzida, adaptada culturalmente e obteve uma validade de conteúdo. Com isso, foi elaborado um folheto para ser distribuído aos pacientes nas consultas, após avaliação dos pés. ${ }^{28}$

Cabe ressaltar que a validação de um instrumento não termina quando se conclui o primeiro estudo. A confiabilidade e a validade variam de acordo com as circunstâncias, população, tipo e finalidade do estudo. ${ }^{17}$ Trata-se de um processo contínuo de avaliação pelo uso continuado do instrumento.

\section{Conclusão}

O instrumento americano: "How to do a 3-minute diabetic foot exam", para avaliação de pacientes diabéticos na Atenção Primária à Saúde, foi traduzido para a língua portuguesa, adaptado ao contexto cultural brasileiro e pelos testes das propriedades de medidas mostrou-se reprodutível, apresentando validade de face, de conteúdo e de construto. Encontra-se disponível para uso através do link: https://osf. io/zfu64/ ou DOI: $10.17605 / O S F . I O / Z F U 64 .^{24}$ 


\section{Agradecimentos}

À Prof. Dra. Maria José Azevedo de Brito Rocha, professora do Mestrado de Ciências Aplicadas à Saúde da UNIVAS, pela sua contribuição na Metodologia do trabalho.

Ao Prof. Dr. Marcos Mesquita Filho, professor do Mestrado de Bioética da UNIVAS, por sua contribuição na análise estatística do estudo.

À Ana Laura Batista Coelho, acadêmica do curso de Medicina, pela contribuição nas ilustrações.

Às Residente de Medicina de Família e Comunidade, Drielly Legati Sarto e Thamara Pereira David Sarmento pelas contribuições na coleta de dados.

\section{Contribuição dos autores}

Concepção ou delineamento do trabalho: MLRMB e BBM. Aquisição, análise ou interpretação dos dados: MLRMB e BBM. Elaboração do rascunho do trabalho e sua revisão crítica para conteúdo intelectual importante: MLRMB e BBM. Aprovação final da versão a ser publicada: MLRMB e BBM. As autoras concordam em prestar contas de todos os aspectos do trabalho, assegurando que as questões relacionadas à acurácia ou integridade de qualquer parte do trabalho sejam devidamente investigadas e resolvidas.

\section{Referências}

1. Oliveira AF, De Marchi ACB, Leguisamo CP, Baldo GV, Wawginiak TA. Estimativa do custo de tratar o pé diabético, como prevenir e economizar recursos. Rev Ciênc Saúde Coletiva. 2014;19(6):1663-71.

2. Artilheiro MMVSA, Franco SC, Schulz VC, Coelho CC. Quem são e como são tratados os pacientes que internam por diabetes mellitus no SUS? Saúde Debate. 2014;38(101):210-24.

3. Word Health Organization (WHO). Diabetes [Internet]. Geneva:WHO; 2016 [acesso 2016 Dez 15]. Disponível em: http://www.who.int/ mediacentre/factsheets/fs312/en/

4. Consenso Internacional sobre Pé Diabético (CIPD). Documento preparado pelo "Grupo de Trabalho Internacional sobre Pé Diabético" [Internet]. Brasília: Secretaria de Estado do Distrito Federal; 2001 [acesso 2017 Abr 2]. Disponível em: http://189.28.128.100/dab/docs/ publicacoes/geral/conce_inter_pediabetico.pdf

5. Mendonça SS, Morais JS, Moura MCGG. Proposta de um protocolo de avaliação fisioterapêutica para os pés de diabéticos. Fisioter Mov. 2011;24(2):285-98.

6. Santos ICRV, Bezerra GC, Souza CL, Pereira LC. Pé diabético: apresentação clínica e relação com o atendimento na atenção básica. Rev Rene. 2011;12(2):393-400.

7. Amaral Júnior AH, Amaral LAH, Bastos MG, Nascimento LC, Alves MJM, Andrade MAP. Prevenção de lesões de membros inferiores e redução da morbidade em pacientes diabéticos. Rev Bras Ortop. 2014;49(5):482-7.

8. Alonso-Fernández M, Mediavilla-Bravo JJ, López-Simarro F, Comas-Samper JM, Carramiñana-Barrera F, Mancera-Romero J, et al. Evaluación de la realización del cribado del pie diabético en Atención Primaria. Endocrinol Nutr. 2014;61(6):311-7.

9. Ministério da Saúde (BR), Secretaria de Atenção à Saúde, Departamento de Atenção Básica. Estratégias para o cuidado da pessoa com doença crônica: Diabetes mellitus. Brasília (DF): Ministério da Saúde; 2013.

10. Andrade NHS, Sasso-Mendes KD, Faria HTG, Martins TA, Santos MA, Teixeira CRS, et al. Pacientes com diabetes mellitus: cuidados e prevenção do pé diabético em atenção primária à saúde. Rev Enferm UERJ (Rio de Janeiro). 2010;18(4):616-21.

11. Ministério da Saúde (BR), Secretaria de Atenção à Saúde, Departamento de Atenção Básica. Manual do pé diabético: estratégias para o Cuidado da Pessoa com Doença Crônica. Brasília (DF): Ministério da Saúde; 2016.

12. Laurindo MC, Recco DC, Roberti DB, Rodrigues CDS. Conhecimento das pessoas diabéticas acerca dos cuidados com os pés. Arq Ciênc Saúde. 2005;12(2):80-4. 
13. American Diabetes Association (ADA). Microvascular complications and foot care. Diabetes Care. 2015;38(Suppl 1):S58-66.

14. Beaton DE, Bombardier C, Guillemin F, Ferraz MB. Guidelines for the process of cross-cultural adaptation of self-report measures. Spine (Phila Pa 1976). 2000;25(24):3186-91.

15. Guillemin F, Bombardier C, Beaton D. Cross-cultural adaptation of health-related quality of life measures: literature review and proposed guidelines. J Clin Epidemiol. 1993;46(12):1417-32.

16. Alexandre NMC, Coluci MZO. Validade de conteúdo nos processos de construção e adaptação de instrumentos de medidas. Ciênc Saúde Coletiva. 2011;16(7):3061-8.

17. Souza AC, Alexandre NMC, Guirardello EB. Propriedades psicométricas na avaliação de instrumentos: avaliação da confiabilidade e da validade. Epidemiol Serv Saúde. 2017;26(3):649-59.

18. Miller JD, Carter E, Shih J, Giovinco NA, Boulton AJ, Mills JL, et al. How to do a 3-minute diabetic foot exam. J Fam Pract. 2014;63(11):646-56.

19. Boulton AJ, Armstrong DG, Albert SF, Frykberg RG, Hellman R, Kirkman MS, et al.; American Diabetes Association; American Association of Clinical Endocrinologists. Comprehensive foot examination and risk assessment: a report of the task force of the foot care interest group of the American Diabetes Association, with endorsement by the American Association of Clinical Endocrinologists. Diabetes Care. 2008;31(8):1679-85.

20. Mokkink LB, Terwee CB, Patrick DL, Alonso J, Stratford PW, Knol DL, et al. The COSMIN study reached international consensus on taxonomy, terminology, and definitions of measurement properties for health-related patient-reported outcomes. J Clin Epidemiol. 2010;63(7):737-45.

21. Terwee CB, Bot SD, de Boer MR, van der Windt DA, Knol DL, Dekker J, et al. Quality criteria were proposed for measurement properties of health status questionnaires. J Clin Epidemiol. 2007;60(1):34-42.

22. Hair Junior JF, Black WC, Babin BJ, Anderson RE, Tatham RL. Análise multivariada de dados. 6 $6^{\text {th }}$ ed. Porto Alegre: Bookman; 2009.

23. Santos HC, Ronsoni MF, Colombo BS, Oliveira CSS, Hohl A, Coral MHC, et al. Escores de neuropatia periférica em diabéticos. Rev Soc Bras Clin Med. 2015;13(1):40-5.

24. Repositório OSF. Como fazer exame de pé diabético em 3 minutos [Internet]. [acesso 2019 Set 10]. Disponível em: https://osf.io/zfu64/. DOI: $10.17605 / O S F . I O / Z F U 64$

25. Amaral AB, Rider EA, Lajolo PP, Tone LG, Pinto RM, Lajolo MP, et al. Development of a Brazilian Portuguese adapted version of the Gap-Kalamazoo communication skills assessment form. Int J Med Educ. 2016;7:400-5.

26. Rayman G, Vas PR, Baker N, Taylor CG Jr, Gooday C, Alder Al, et al. The Ipswich Touch Test: a simple and novel method to identify inpatients with diabetes at risk of foot ulceration. Diabetes Care. 2011;34(7):1517-8.

27. Pinto JS, Lopes MJ, Oliveira JV, Amaro JP, Costa LD. Métodos para estimação de reprodutividade de medidas [Internet]. Porto: Faculdade de Medicina do Porto; 2017 [acesso 2017 Abr 10]. Disponível em: https://users.med.up.pt/ joakim/intromed/coeficientecorrelacaointraclasse.htm

28. Pereira DA, Costa NMSC, Sousa ALL, Jardim PCBV, Zanini CRO. Efeito de intervenção educativa sobre o conhecimento da doença em pacientes com diabetes mellitus. Rev Lat Am Enferm. 2012;20(3):478-85. 